\title{
Higher proportion of low-virulence anaerobic bacterial infection in young patients with intervertebral disc herniation
}

\author{
GUOQING TANG ${ }^{1,2^{*}},{\text { YONG } \mathrm{CHEN}^{2,3^{*}}, \mathrm{JI} \mathrm{CHEN}^{2}, \mathrm{ZHUO}_{\mathrm{WANG}}^{2} \text { and WEIMIN JIANG }}^{1}$ \\ ${ }^{1}$ Orthopedic Center, The First Affiliated Hospital of Soochow University, Suzhou, Jiangsu 215000; \\ ${ }^{2}$ Orthopedic Center, Kunshan Hospital of Traditional Chinese Medicine, Suzhou, Jiangsu 215300; \\ ${ }^{3}$ Department of Orthopaedics, The Second Hospital Affiliated to Soochow University, Suzhou, Jiangsu 215008, P.R. China
}

Received February 7, 2019; Accepted July 29, 2019

DOI: $10.3892 /$ etm.2019.7910

\begin{abstract}
Intervertebral disc (IVD) degeneration caused by the latent infection of low-virulence anaerobic bacteria (LVAB) is a hot research topic. The present study analyzed and compared the positive rate of LVAB within IVDs of patients of different ages. IVD samples were intraoperatively retrieved from 176zpatients with disc herniation and subjected to microbiological culture. Subsequently, all the patients were classified into 3 groups based on their ages as follows: Group A (age <30 years), group B (age from 30 to 50 years) and group $C$ (age $>50$ years) and the positive rates of LVAB were compared among the three groups. The severity of IVD degeneration was also assessed by measuring intervertebral height. Of the 176 cultured discs, 39 samples had bacterial growth, while 6 of them were suspiciously contaminated. Follwoing the exclusion of the 6 suspicious samples, 31 samples were Propionibacterium acnes and 2 samples were coagulase-negative Staphylococci of the remaining 33 samples, The bacterial positive rates were significantly higher in younger patients, with $34.4 \%(11 / 32), 25.5 \%$ (13/51) and $10.3 \%$ (9/87) in group A, B and C, respectively. More importantly, the bacterial-positive samples had a significantly lower disc height compared with the negative samples in groups A and $\mathrm{B}$. Therefore, it can be reasonably concluded that younger patients have a much higher prevalence of LVAB infection in herniated IVDs and a greater severity of IVD degeneration when infected by bacteria. The LVAB may have a strong association with IVD degeneration, particularly in young patients.
\end{abstract}

Correspondence to: Dr Weimin Jiang, Orthopedic Center, The First Affiliated Hospital of Soochow University, 188 Shizi Street, Suzhou, Jiangsu 215000, P.R. China

E-mail: jiangwm1967@163.com

${ }^{*}$ Contributed equally

Key words: low-virulence anaerobic bacteria, Propionibacterium acnes, coagulase-negative Staphylococci, disc herniation, young patient

\section{Introduction}

Intervertebral disc (IVD) degeneration is the initiation of a series of spinal diseases, such as disc herniation, degenerative spondylolisthesis and spinal stenosis and is associated with a number of symptoms, such as lower back pain, neck pain or sciatica (1). A number of factors contribute to the pathological process of IVD degeneration, such as age, trauma and over-load stress $(1,2)$. However, there is still no exact etiology to explain the process of IVD degeneration.

The peak incidence of disc degeneration occurs among middle-aged or older patients; however, a recent epidemiological study demonstrated that an increasing number of young patients (age, $<45$ years) also suffer from disc degeneration or disc herniation (3). However, the reasons for disc degeneration or herniation in young patients remain unclear. Therefore, this poses a challenge for all spinal surgeons and researchers worldwide.

Apart from mechanical factors or age, accumulating evidence has indicated that low-virulence anaerobic bacteria (LVAB) are a new pathogen involved in IVD degeneration. The study by Stirling et al (4) first reported that the prevalence of Propionibacterium acnes (P. acnes) was as high as 53\% in the herniated IVDs of patients. Furthermore, a systematic review concluded that LVAB played a critical role in disc herniation with lower back pain and that bacterial presence was related to Modic changes associated with disc herniation (5). In addition, animal studies have confirmed that the inoculation of $P$. acnes causes or deteriorates the degeneration of IVDs in rats or rabbits $(6,7)$. Therefore, the pathological role of LVAB in IVD degeneration or herniation warrants further attention.

In this study, the prevalence of LVAB in herniated IVDs was first analyzed. The present study hypothesized that latent infection with LVAB may be a critical risk factor for disc degeneration in young patients. The present study proposes a new hypothesis regarding IVD degeneration in young patients and this may lead to the development of novel therapeutic strategies for IVD in the future.

\section{Materials and methods}

Patients. From January 2017 to January 2018, 176 consecutive patients with single level IVD herniation were enrolled in this 
study. The patient characteristics are shown in Table I. All of these patients suffered from severe lower back pain and/or sciatica for several months and conservative treatment was non-effective for all of them. IVD samples were collected and analyzed when the patients underwent discectomy or discectomy combined with instrumented posterior lumbar fusion. The collection of samples was authorized by the Committee on Ethics and the Institutional Review Board of Soochow University and each patient signed written informed consent forms.

Among the collected IVD samples, 33 were the level of L3-L4, 95 were L4-L5 and 48 were L5-S1. None of the patients had a history of antibiotics administration one month prior to the surgery and none had any clinical or biological signs of infection.

Preoperative imaging evaluation. To evaluate the severity of IVD degeneration, intervertebral height was measured using the protocol reported by Frobin et al (8) and Zhou et al (9). In brief, lateral X-ray films in the standing position were obtained prior to surgery. Subsequently, a line connecting the midpoint of ventral margin and dorsal margin of the vertebrae was drawn. The line was defined as the midplane. An angle between two adjacent vertebrae was measured between their midplanes. The ventral height of a lumbar disc was then calculated as the sum of the perpendicular distances of the corner of the cranial vertebra and the corner of the caudal vertebra from the bisectrix between the two midplanes. To obtain a more accurate intervertebral height which is independent of the angle of lumbar lordosis, a correction was applied to convert disc height measured in arbitrary angles to height at standard angles according to the method proposed by Frobin et al (8).

Intraoperative samples. According to the protocol of the Institutional Review Board, all patients had to receive a single dose of $2 \mathrm{~g}$ cefazolin for prophylactic antibiotic treatment during surgery. To avoid possible contamination during tissue harvest, the incision was disinfected at least twice with povidone iodine. Only fresh instruments were then used for the handling of the samples. The harvested IVD samples were rapidly stored in sterilized tubes and transported to the laboratory of bacteriology. Finally, as contamination markers, some muscle and ligaments surrounded the IVDs were collected at the same time during surgery.

Microbiological culture. The collected samples were cultured under a class II laminar flow safety cabinet at the laboratory of bacteriology in The First Affiliated Hospital of Soochow University. Briefly, the harvested IVD samples were dissected into 5 sections and 3 of these were split and embedded into the plates, while the remaining 2 segments were placed into broth. The three segments were embedded into MacConkey agar plates for aerobic culture, chocolate agar plates in $5 \% \mathrm{CO}_{2}$ and horse blood agar plates for aerobic incubation, respectively. All plates were incubated for at least 14 days at $37^{\circ} \mathrm{C}$ and the results were examined on the 7 th and 14th day.

The broth used in this study was cooked meat medium broth. One segment was first enriched for $48 \mathrm{~h}$ at $37^{\circ} \mathrm{C}$ and then transferred to horse blood agar plates for anaerobic incubation and then onto chocolate agar plates in $5 \% \mathrm{CO}_{2}$ at $37^{\circ} \mathrm{C}$ for 7 days, respectively. The other segment was cultured in broth for 7 days and then anaerobically cultured on horse blood agar plates at $37^{\circ} \mathrm{C}$ for 7 days and with chocolate agar plates under $5 \% \mathrm{CO}_{2}$ at $37^{\circ} \mathrm{C}$ for 7 days. If there was any bacterial growth in the broth or plates, the bacteria were collected and identified with the 16S rDNA method according to the previously described technique (9). The method of culturing the control samples was the same as described for the culture of IVDs.

Statistical analysis. SPSS 22.0 software (IBM Corp.) was used for statistical analysis of data. The continuous data are expressed as the mean \pm standard deviation. When there were two groups, an unpaired two-sided Student's t-test was used for analysis. In addition, for the analysis of categorical variables, the Chi-Square test was conducted. $\mathrm{P}<0.05$ was considered to indciate a statistically significant difference. Each experiment was carried out with 3 independent repeats.

\section{Results}

Microbiological culture results of disc samples. A total of 176 IVD samples were harvested for microbiological culture and 39 disc samples $(39 / 176,22.1 \%)$ yielded positive results. Among the 39 cases, 6 were considered as possible contamination due to fact that the contamination markers of muscle and ligaments were positive for bacteria at the same time and they were excluded in the ensuing analysis (details in Supplementary Table SI). The remaining 33 samples (33/176, $18.7 \%$ ) exhibited bacterial growth only in the discs and they were considered to be bacteria-positive samples. Out of the 33 samples, $P$. acnes was detected in 31 samples $(31 / 176,17.6 \%)$ and coagulase-negative Staphylococcus (CNS) was found in 2 samples $(2 / 176,1.1 \%)$.

With the further analysis of the epidemiological data, a significant difference was demonstrated in the age between the patients with or without bacteria in IVDs $(\mathrm{P}=0.001$; Table I). However, no significant difference was observed in sex, smoking status, diabetes and the levels of discs between the positive and negative patients (Table I).

Higher proportion of bacterial infection in younger patients. Due the statistically significant difference in age, all patients (excluding the 6 suspicious cases) were further divided into three subgroups based on age for analysis as follows: Group A (age, $<30$ years), group B (age, 30 to 50 years) and group C (age, $>50$ years). In group A, 10 patients had IVDs infected by $P$. acnes and one sample infected by CNS. In group B, 12 IVDs were infected by $P$. acnes and one IVD was infected by CNS. For group C, P. acnes was found in all of the 9 samples. The proportion of LVAB infection in each group was $34.4 \%$ (11/32), $25.5 \%$ (13/51) and $10.3 \%$ (9/87), respectively and there was a statistically significant difference between the three groups ( $\mathrm{P}=0.005$; Table II). The rates of bacterial infection were significantly increased in the IVDs of younger patients. The distribution of bacterial-positive and bacterial-negative subjects by age is demonstrated in Fig. SI.

Infection of bacteria with more severe disc degeneration. The disc height was compared between the bacterial-positive and 
Table I. Patient and clinical characteristics association with bacterial presence.

\begin{tabular}{|c|c|c|c|c|}
\hline Parameters & $\mathrm{n}=170^{\mathrm{a}}$ & Positive samples $n=33^{a}$ & Bacteria-free samples $n=137$ & P-value \\
\hline Age & $51.69 \pm 15.38$ & $46.73 \pm 16.54$ & $55.74 \pm 13.35$ & 0.001 \\
\hline Male & 92 & 21 & 71 & 0.221 \\
\hline Female & 78 & 12 & 66 & \\
\hline Diabetes & 43 & 9 & 34 & 0.770 \\
\hline Non-diabetic & 127 & 24 & 103 & \\
\hline Smoker & 69 & 17 & 52 & 0.154 \\
\hline Non-smoker & 101 & 16 & 85 & \\
\hline \multicolumn{5}{|l|}{ Level of IVDs } \\
\hline $\mathrm{L} 3 / 4$ & 31 & 8 & 23 & 0.144 \\
\hline $\mathrm{L} 4 / 5$ & 93 & 13 & 80 & \\
\hline $\mathrm{L} 5 / \mathrm{S} 1$ & 46 & 12 & 34 & \\
\hline
\end{tabular}

${ }^{\mathrm{a}} \mathrm{A}$ total of 6 cases of suspicious contamination were excluded. IVDs, intervertebral discs.

Table II. Proportion of bacterial infection in each group at different ages.

\begin{tabular}{lccc}
\hline & \multicolumn{2}{c}{ Culture on discs } & \\
\cline { 2 - 5 } & Positive culture & Bacteria free & Total \\
\hline Group A $($ Age $<30)$ & $11(6.5 \%)$ & $21(12.4 \%)$ & 32 \\
Group B $(30 \leq$ Age $\leq 50)$ & $13(7.6 \%)$ & $38(22.4 \%)$ & 51 \\
Group C $($ Age $>50)$ & $9(5.3 \%)$ & $78(45.8 \%)$ & 87 \\
Total & $33^{\mathrm{a}}$ & 137 & $170^{\mathrm{a}}$ \\
\hline
\end{tabular}

${ }^{\mathrm{a} A}$ total of 6 cases of suspicious contamination were excluded.

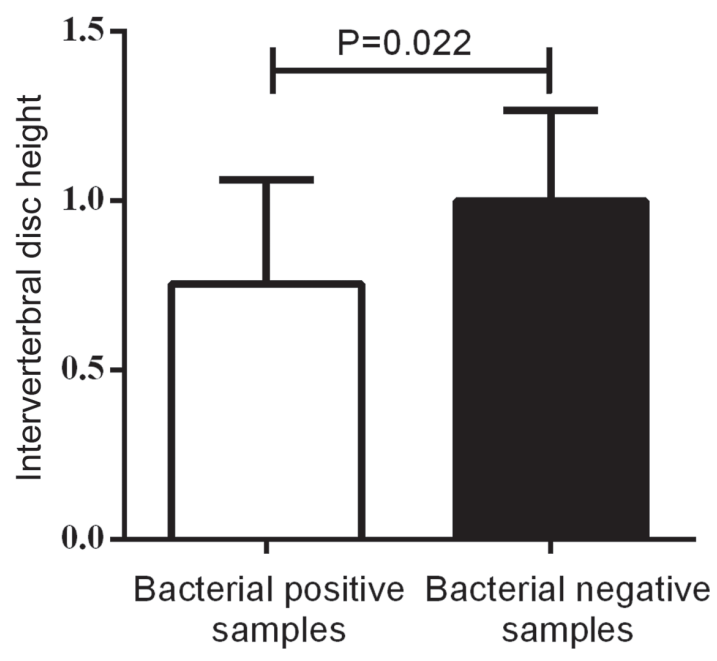

Figure 1. Comparison of intervertebral disc height between bacterial-positive samples and bacteria-free samples. When comparing the two groups, the bacterial-positive samples had a lower intervertebral height than the bacteria-free samples. A two-sided Student's t-test was conducted and the data are expressed as the mean \pm standard deviation.

bacterial-negative samples. As depicted in Fig. 1, the positive samples had a significantly lower disc height than that of the bacterial-negative samples $(\mathrm{P}=0.022)$. When comparing the disc height in each group, the disc height was significantly lower in the bacterial-positive samples than that of the negative samples in groups $\mathrm{A}$ and $\mathrm{B}$, while there was no statistically significant difference in group $\mathrm{C}(\mathrm{P}=0.003$ and $\mathrm{P}=0.023$, respectively; Fig. 2). This suggested that low virulence bacterial infection may play a critical role in the IVD degeneration of young patients.

\section{Discussion}

There is increasing evidence to suggest that LVAB may be a novel etiological factor for a series of spinal diseases and contributes to plenty of symptoms, such as sciatica, neck pain and back pain (10). In this study, it was reported that $22.1 \%$ patients had LVAB in the IVDs, which was in the range of $8-53 \%$ according to a previous study (10). Contamination markers were also established during tissue harvest and culture following a previously published protocol (9); therefore, suspiciously contaminated cases were excluded in this study. Therefore, the prevalence of LVAB was $18.7 \%$ in herniated IVDs after the exclusion of six suspicious cases.

Of note, in this study, it was revealed that the proportion of LVAB infection was much higher in younger patients. 


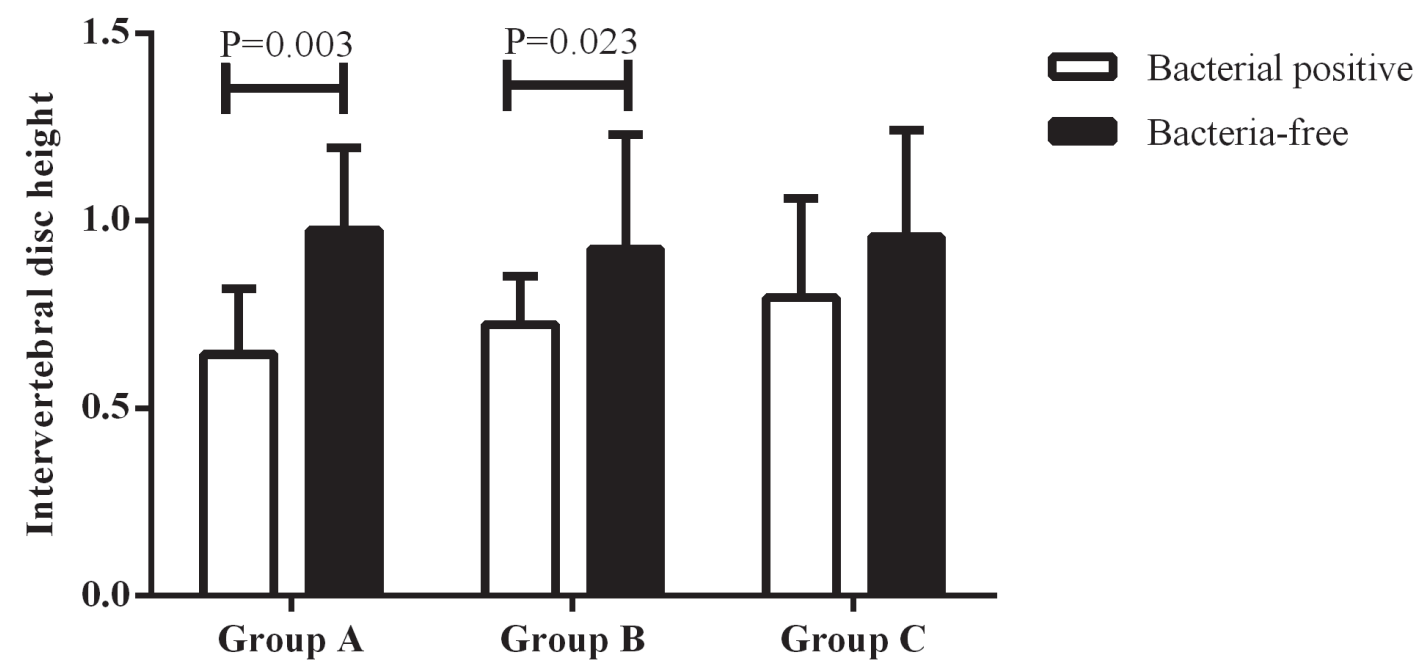

Figure 2. Comparison of intervertebral disc height between bacterial-positive samples and bacterial-free samples among each age group. Among the three groups, the bacterial-positive samples had a lower intervertebral height than the bacterial-free samples in patients with aged $<30$ years and aged from 30 to 50 years. A two-sided Student's t-test was conducted and the data reexpressed as the mean \pm standard deviation.

Conventional viewpoints always considered that age and excessive load are the key factors that induce IVD degeneration (2), however, it is hard to explain the degeneration of IVDs in young patients with these two factors. In this study, it was found that there was a significant difference in that young patients had a higher rate of bacterial infection in the IVDs, which highly suggested that the LVAB, particularly $P$. acnes, may be one of the critical factors for IVD degeneration in young patients, as previous studies have proven that $P$. acnes is a definite factor responsible for the deterioration of IVD degeneration $(9,11,12)$. In addition, patients with bacterial infection had a higher severity of IVD degeneration than that of negative patients, further demonstrating that bacteria may deteriorate IVD degeneration.

A number of studies have suggested the $P$. acnes accelerates the degeneration of IVDs. For example, Lin et al (11) and Zhou et al (9) demonstrated that the patients with $P$. acnes-positive IVDs had a lower intervertebral disc height, which proved that infection with $P$. acnes in IVDs is a novel risk factor that deteriorates disc degeneration. Moreover, the inoculation of $P$. acnes into the IVDs of rats or rabbits has been shown to cause severe disc degeneration when compared with the control samples $(6,7)$. Thus, it can be reasonably concluded that $P$. acnes is a novel etiological factor for IVD degeneration.

Nevertheless, it is hard to explain why young patients had a higher prevalence of low-virulence anaerobic bacteria, particularly $P$. acnes, in IVDs. To date, there is still no theory or hypothesis to explain how $P$. acnes latently infects IVDs. Albert et al (13) hypothesized that the $P$. acnes enters the circulatory system via the breaking of the skin or mucous membranes and then invades the IVDs through the tear of the anulus fibrosus. However, the study by Li et al (12) did not support this hypothesis, as there was no bacterial growth in the degenerated IVDs following the injection of $P$. acnes into the veins of rabbits for several days. The present study therefore hypothesized that the IVDs harvested from younger patients may be relatively less degenerated than that from older patients, so the younger IVDs may provide a more suitable environment for the survival and reproduction of LVAB. Therefore, the rates of LVAB infection in IVDs may be higher in younger patients. In the future, further studies are required to explore the exact mechanisms involved.

However, several limitations still exist in this study. Firstly, the reason why young patients had a higher prevalence of $P$. acnes infection remains unclear. More evidence obtained from clinical or animal studies is required to solve this matter. In addition, the preoperative administration of antibiotics may reduce the bacterial positive rates in the IVDs under the protocol of the Institutional Ethics Board. Finally, a greater number of patients have to be analyzed in the future for a more accurate prevalence.

In conclusion, this study reported that the prevalence of LVAB was $18.7 \%$ in IVDs and younger patients had much higher positive rates of bacterial infection and a greater severity of IVD degeneration. Therefore, LVAB may play a crucial role in the pathophysiology of intervertebral disc herniation, particularly in younger patients.

\section{Acknowledgements}

Not applicable.

\section{Funding}

The present study was supported by grants from the Natural Science Foundation of Jiangsu Province (grant no. BK20161274), the Natural Science Foundation of Suzhou (grant no. kjxw2015056) and the Science and Technology Bureau of Kunshan (grant no. KS1547).

\section{Availability of data and materials}

The datasets used and/or analyzed during the current study are available from the corresponding author on reasonable request. 


\section{Authors' contributions}

The manuscript was approved and contributed to by all authors. WMJ conceived and designed the study; GQT acquired the data, interpreted the results and was involved in drafting and revising the manuscript; YC performed the radiological analysis, the bacterial culture and interpreted the results; JC collected and transported the samples, acquired the epidemiological data of patients and interpreted the results; ZW performed the statistical analysis and interpreted the results during drafting and revising the manuscript.

\section{Ethics approval and consent to participate}

This study was approved by the Committee on Ethics and the Institutional Review Board of Soochow University and each patient signed written informed consent forms.

\section{Patient consent for publication}

Written informed consent for publication was obtained from all individual participants included in the study.

\section{Competing interests}

The authors declare that they have no competing interests.

\section{References}

1. Modic MT and Ross JS: Lumbar degenerative disk disease. Radiology 245: 43-61, 2007.

2. Zhang YG, Sun Z, Zhang Z, Liu J and Guo X: Risk factors for lumbar intervertebral disc herniation in Chinese population: A case-control study. Spine (Phila Pa 1976) 34: E918-E922, 2009.

3. Kim YK, Kang D, Lee I and Kim SY: Differences in the incidence of symptomatic cervical and lumbar disc herniation according to age, sex and national health insurance eligibility: A pilot study on the Disease's association with work. Int J Environ Res Public Health 15: pii: E2094, 2018.
4. Stirling A, Worthington T, Rafiq M, Lambert PA and Elliott TS: Association between sciatica and Propionibacterium acnes. Lancet 357: 2024-2025, 2001.

5. Urquhart DM, Zheng Y, Cheng AC, Rosenfeld JV, Chan P, Liew S, Hussain SM and Cicuttini FM: Could low grade bacterial infection contribute to low back pain? A systematic review. BMC Med 13: 13, 2015.

6. Chen Z, Zheng Y, Yuan Y, Jiao Y, Xiao J, Zhou Z and Cao P. Modic changes and disc degeneration caused by inoculation of Propionibacterium acnes inside intervertebral discs of rabbits: A pilot study. Biomed Res Int 2016: 9612437, 2016.

7. Dudli S, Liebenberg E, Magnitsky S, Miller S, Demir-Deviren S and Lotz JC: Propionibacterium acnes infected intervertebral discs cause vertebral bone marrow lesions consistent with Modic changes. J Orthop Res 34: 1447-1455, 2016.

8. Frobin W, Brinckmann P, Kramer M and Hartwig E: Height of lumbar discs measured from radiographs compared with degeneration and height classified from MR images. Eur Radiol 11: 263-269, 2001

9. Zhou Z, Chen Z, Zheng Y, Cao P, Liang Y, Zhang X, Wu W, Xiao J and Qiu S: Relationship between annular tear and presence of Propionibacterium acnes in lumbar intervertebral disc. Eur Spine J 24: 2496-2502, 2015.

10. Chen Z, Cao P, Zhou Z, Yuan Y, Jiao Y and Zheng Y: Overview: The role of Propionibacterium acnes in nonpyogenic intervertebral discs. Int Orthop 40: 1291-1298, 2016.

11. Lin Y, Jiao Y, Yuan Y, Zhou Z, Zheng Y, Xiao J, Li C, Chen Z and $\mathrm{Cao}$ P: Propionibacterium acnes induces intervertebral disc degeneration by promoting nucleus pulposus cell apoptosis via the TLR2/JNK/mitochondrial-mediated pathway. Emerg Microbes Infect 7: 1, 2018.

12. Li B, Dong Z, Wu Y, Zeng J, Zheng Q, Xiao B, Cai X and Xiao Z: Association between lumbar disc degeneration and Propionibacterium acnes infection: Clinical research and preliminary exploration of animal experiment. Spine (Phila Pa 1976) 14: E764-E769, 2016.

13. Albert HB, Kjaer P, Jensen TS, Sorensen JS, Bendix T and Manniche C: Modic changes, possible causes and relation to low back pain. Med Hypotheses 70: 361-368, 2008.

(i) $(-)$ This work is licensed under a Creative Commons

CY NC ND Attribution-NonCommercial-NoDerivatives 4.0 International (CC BY-NC-ND 4.0) License. 